\title{
Antimicrobials in treatment of periodontal disease -A review
}

\author{
Dr Preethe Paddmanabhan* \\ Sr Lecturer Department of Periodontics Sree balaji dental college \& Hospital,Chennai. INDIA
}

\begin{abstract}
Periodontitis is a multifactorial disease that have been associated with multiple etiological factors. These disorders are as a result of accumulation of dental plaque \& clinical signs are caused by resultant inflammatory response. However complete removal of biofilm is not always possible by scaling \& root planing alone. Reinfection of periodontal pockets results from residual biofilm and penetration of bacteria in the connective tissue needs the combination of Scaling and root planing \& antimicrobial therapy. The Knowledge about the biomechanism of advantage and disadvantage of antimicrobial agents is essential in successful management \& prevention of periodontal disease.
\end{abstract}

Key words: Antimicrobial agents, antibiotics, antiseptics, local drug delivery, Host modulation therapy

\section{Introduction}

During early days the resolution of inflammation was either by spontaneous in nature by exfoliation or by extraction. By the turn of 20th century, it was considered that calculus was a mechanical irritant $\&$ its removal by scaling \& root planning was practiced. Modern era of periodontal therapy began roughly 40 years ago, where surgical pocket elimination \& osseous surgery was performed. In 1960 \& 1970 's infectious nature of periodontitis has become apparent $\&$ this concept leads to the use of antimicrobials $\&$ anti inflammatory drugs for periodontal infections. Later regenerative procedures has come out for the treatment of periodontal disease.

According ADA Journal of American dental association 2006 emphases the importance of use of antimicrobial rinses as a part of daily oral care regimen to control the accumulation of dental plaque.

Thomas \& Nakaishi 2006 provide a overview of understanding periodontal microbiology \& concept of dental plaque as a biofilm. Numerous studies are cited that demonstrate the efficacy of antimicrobial agent in reducing plaque \& microbiota

According to Michael G Jorgensen et al 2000,a multifaceted yet straight forward approach is presented for management of destructive periodontal disease. By considering bacterial specificity in periodontitis, various therapeutic modalities are available to identify, suppress and eradicate periodontal pathogens. This will lead to a scientifically based treatment plan. By appreciating the advantage \& usefulness of antimicrobials agents is very much essentially in preventing periodontal disease. The intent of this article is to review the various antimicrobials in treatment of periodontal disease.

\section{Risk factors}

It is been suggested that severity of periodontal disease, its rate of progression, and its response to therapy vary from patient to patient. Pathogenic bacteria essential for initiation of the disease but insufficient by themselves to cause the disease. The host may be susceptible, and its patient's risk factor that determine the susceptibility to the disease.

\section{GENETIC RISK FACTORS}

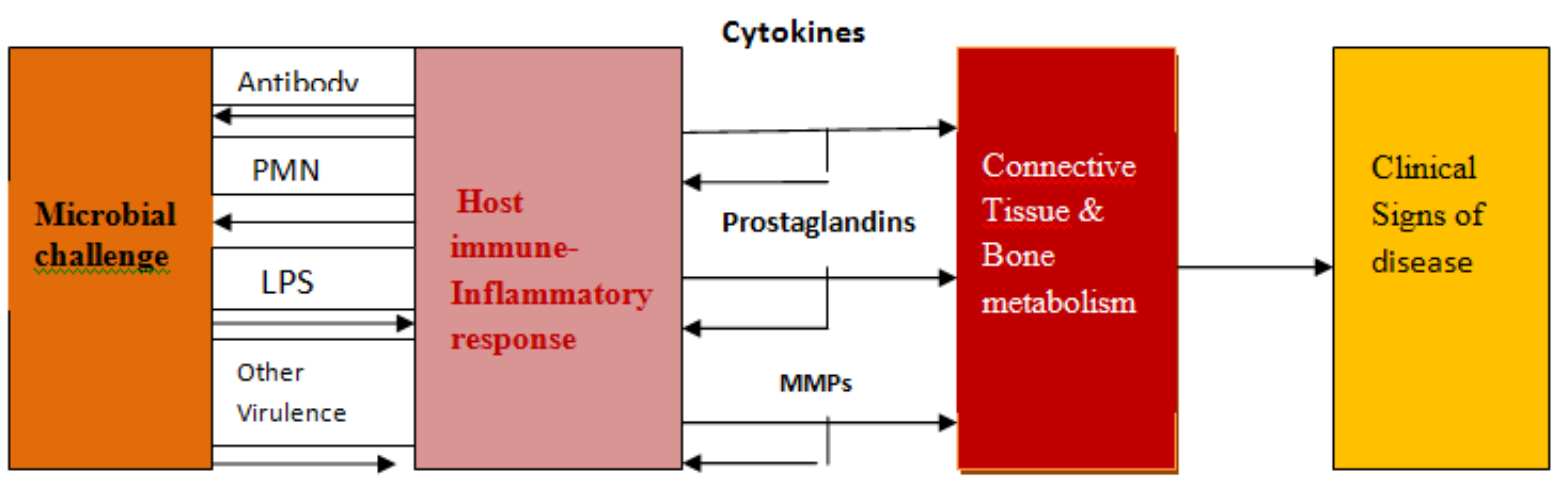




\section{Risk Factors}

Risk Factors may be environmental, behavioral or biological factors that when present, increase the likelihood that an individual will develop the disease. Risk factors are identified through longitudinal studies of patients with disease interest.

Exposure to risk factor or factors may occur at single point of time or continuously.

Risk indicators are probable or putative risk factors that can be indentified in cross sectional studies but not confirmed through longitudinal studies.

Biofilm: A Biofilm is a well organized, cooperating community of bacteria that adhere to surfaces and is embedded in a extracellular slime layer(1). Once a bacterium attaches to the surface, it activates a whole different set of genes that gives the bacterium different characteristics from those that it had as a free floating organism. It has been estimated that more than $99 \%$ of all bacteria on the earth live as attached bacteria (2). Biofilm can be found on medical and dental implants living in intravenous and urinary catheters, contact lenses, prosthetic devices such as heart valves, biliary stents, pace makers and artificial joints.

Mechanical debridement of the dental biofilm and elimination of local irritating factors are the basis of intial periodontal therapies. Longitudinal studies have been demonstrated the effectiveness of this approach, which is based on scaling, Root planning, reinforcement of patient oral hygiene practices and regular follow up to eliminate new deposits $(3,4)$. The concept of antibiotic periodontal therapy revolves around the drug, the pathogenic microorganisms and the host.

\section{Systemic Antibiotics}

Systemic antibiotics therapy for periodontal treatment usually involves monotherapy based on the $\beta$ lactams, including amoxicillin with or without clavulanic acid, metronidazole, tetracyclines (tetracycline, doxycycline, minocycline), clindamycin and ciprofloxacin (5).

\section{INDICATIONS FOR ANTIBIOTICS IN PERIODONTAL THERAPY}

- In accordance with the general principles of prescribing antibiotics, however, it is essential that the drugs are administered only after careful case selection, and antibiotic therapy should be a substitute for the routine and time-honored treatment regimens.

The following periodontal disease states would justify the adjunctive use of antibiotics:

1. In severe cases both of acute necrotizing ulcerative gingivitis and periodontitis, especially if there are signs of systemic involvement, metronidazole can quickly alleviate the symptoms, which then permitsthroughmechanical debridement to be carried out.

2. Occasionally, the local infection of a periodontal abscess can spread within tissue planes to cause marked facial swelling and systemic involvement. In these cases, broad-spectrum antibiotics should be prescribed to control the infection. Careful clinical and radiographic examinations must be done to establish whether the lesion is wholly periodontal in origin or whether there is pulpal involvement of the associated teeth.

3. Multiple abscess formation and gross periodontal infection would necessitate the administration of antibiotics (metronidazole and tetracycline). A number of medical conditions (e.g. Diabetes mellitus) can predispose to advanced periodontal destruction with abscess formation.

4. Antibiotic therapy is warranted in cases of periodontal disease, which, despite through non

Surgical management and good plaque control, continue to show breakdown and loss of attachment. These socalled refractory cases can benefit from a short course of antibiotic therapy. The drug of choice should be determined from sampling the cultivable pocket flora from which the predominant populating organisms can be identified.

5. Antibiotic therapy is recommended in the management of cases of AP either in combination with flap surgery or a non-surgical treatment programme.

Tetracycline was the first antibiotics for treatment of periodontitis that received scientific evaluation. In a split mouth design, Listgarten et al and Hellden et al tested the clinical efficacy of tetracycline in 12 adult periodontitis patient. No statistically significant differences were measured in favor of the tetracycline group, although the mean probing depth and the attachment level improved slightly more in the tetracycline group $(6,7)$. Pencillins are broad class of antibiotics that inhibit bacterial cell wall synthesis and directly result in the death of the cell. B-lactamases are relatively common in periodontal pockets, with the incidence showing a positive correlation with the pocket depth (8).

Magnusson et al reported, for sites deemed clinically active on average $2 \mathrm{~mm}$ gain in clinical attachment 3 months post operative therapy and an average decrease of $2.5 \mathrm{~mm}$ probing pocket depth 6 months post therapy. These results were obtained using 10 patients with the refractory periodontal disease following 
mechanical debridement and the adjunctive use of augmentin for 2 weeks (9)Both gain attachment and decrease in pocket depth were maintained for 1 year.

Clindamycin is bacteriostatic and inhibits bacterial protein synthesis by binding to the 50S ribosomal subunits. The drug is most effective against gram negative anaerobes and is very active against gram negative anaerobes associated periodontal flora(10).

Metronidazole: Metronidazole, a 5- nitroimidazole compound specifically targets anaerobic microorganisms but has essentially no activity against aerobic or microaerophilic bacteria. Cytotoxic metabolites of metronidazole directly interact with the bacterial DNA and possibly other macromolecules, resulting in cell death. Metronidazole crosses placental barrier enter fetal circulatory system. It is also secreted in breast milk so contraindicated in pregnant women. The most common adverse effect of metronidazole involve the gastrointestinal tract. About $12 \%$ of the patient experience nausea which is accompanied by headache, anorexia and vomiting. Drowsiness, depression, skin rashes and vaginal and/or urethral burning have been reported. Metronidazole penetrates GCF $(11,12)$ for most of putative pathogens(13).

Studies by losche et al enrolling adult periodontitis subjects indicated that metronidazole in conjugation with mechanical debridement decreases the number of teeth requiring periodontal surgery or extraction due to periodontitis $(14,15)$

Ciprofloxacin Ciprofloxacin is a quinolone antibiotic and is bactericidal due to its mode of action on bactericidal DNA replication. Generally, ciprofloxacin like most quinolones is well tolerated. When present, drug related side effects are generally nausea, headache, abdominal discomfort or epigastric upset. Other side effects include photosensitivity, dizziness and light headedness. Ciprofloxacin is excreted in human breast milk and may cross the placental barrier. Thus it is contraindicated during lactation and pregnancy. The adjunctive use of metronidazole/ciprofloxacin in periodontal therapy is useful under certain circumstances.

\section{Antiseptics\& Disinfectants}

Chlorhexidine: Chlorhexidine has often been employed as an adjunct to mechanical debridement due to its broad-spectrum antimicrobial activity, substantivity in the oral cavity and ease of use during oral irrigation or gel placement. However, only minor clinical benefits have been obtained and antimicrobial effects on the subgingival microflora were shown to be negligible (16) The biodegradable chlorhexidine containing gelatin chip (Periochip) has received Food \& Drug administration approval in the united states for use as an adjunct to SRP. The gelatin chip is directly placed directly into the periodontal pocket releasing $2.5 \mathrm{mg}$ of chlorhexidine over a period of 7-10 days. Chlorhexidine reached an average concentration equivalent to $125 \mu \mathrm{g}$ per $\mathrm{ml}$ of gingival crevicular fluid, showing significant improvement in the pocket depth relative to SRP alone but no change in Clinical attachment level(17).

Povidone iodine: Povidone iodine (PVP-Iodine) is a bactericidal anti-septic whose mechanism of action include oxidation of amino, thiol and hydroxyl moieties of amino acids and nucleotide. Rosling et al 1986 \& Rosling BG, Slots J et al 1983 has shown additive effects of PVP-iodine irrigation as an adjunt to SRP and improvements in clinical indicies and microbial parameters.

Greenstein (18) in recent review of the effects of PVP-iodine, found evidence indicating PVP-Iodine irrigation, delivered to the periodontal pocket via ultrasonic scalers, achieved better results in deep pockets than water. Greenstein concluded that the literature suggested that PVP- iodine is potentially beneficial in the management of some periodontal disease.

\section{Local Drug Delivery}

Local Drug Delivery of antimicrobial agents allows the use of concentrations up to 100 times higher than when systemic routes of administration are employed

Vehicles that have been employed for sustained delivery in periodontics include pastes, ointments, gels, fibers, strips, spheres, discs and chips. Tetracycline Metronidazole, minocycline, doxycycline, have been used in sustained drug delivery devices. Most local drug delivery systems have been evaluated as adjunctive treatment to scaling and root planing and some additional benefits have been reported for some delivery systems, though most studies are short term in nature. Locally delivered antibiotics have little or no effects on A.actinomy cetemcomitans and other periodontal pathogens invading gingival connective tissue.

Objectives of Local Drug Delivery (Rams et al 1996)(19)

- Achievement and maintenance of therapeutic levels of the drug for the required period of time.

- To inhibit or kill the putative pathogen

- To provide adequate concentration without any harm to the tissues.

- Use of antimicrobials as part of regenerative periodontal therapy (Goodson 1994). Indications

- Where medically compromised patients are contraindicated for surgery. 
- During maintenance phase

- Emergency situations including the treatment of ANUG and post surgical infections.

- As an adjunct with mechanical therapy.

Contraindications

- As a monotherapy

- Allergy to the antimicrobials used

- During pregnancy and lactation

It is necessary to keep in mind that a drug should meet five criteria to be effective in treating disease (Goodson 1979).

- Inhibit or kill the putative pathogen.

- Reach the site.

- Have adequate concentration.

- Be there long enough.

- Do no harm. Periodontal clearance

- GCF is an altered serum Transudate found in the gingival sulcus. GCF flow into periodontal pockets averages $20 \mu \mathrm{l} /$ hour and markedly increased with gingival tissue inflammation. Total pocket fluid volume thus may turn over 40 times an hour in a moderate sized periodontal pocket of $5 \mathrm{~mm}(0.5 \mu \mathrm{l}$ volume), which is more frequent than the oral cavity salivary turnover rate of about 28 times an hour (Goodson, 1989).

\section{Host modulation Therapy}

Therapeutic modalities should aim not only in arresting and preventing the progress of periodontal tissue destruction but also at reestablishing and regenerating the periodontal tissues previously lost to disease. Agents that modulate the host response for periodontal repair and regeneration include, exogenous growth and differentiation factors, attachment factors which enhance the normal wound healing response that may be of insufficient magnitude to promote complete regeneration of all attachment structures.

\section{Role of host response in periodontal disease progression:}

The gram negative bacteria that are associated with the progressive disease are capable of producing various bioactive molecules, that can directly affect the host including lipopolysaccharide(LPS),proteases, and other enzymes and cytotoxic molecules(20). They are capable of triggering host destructive mechanisms that amplify the local destructive effects. The indirect or host mediated tissue destruction effects result in induction, stimulation or activation of host cells or humoral factors

Many of these are immunological processes resulting in the pathological alternation of fibroblast, activation of macrophages, lymphocytes, modulation of fibroblast growth and synthesis of

collagen and bone resorption stimulated by products of activated mononuclear cells such as interleukin 1,prostaglandins, tumour necrosis factor and other endogenous bone resorptive factor. The interaction between the host cells and molecules produced by the bacteria determine the outcome of infection

Goodson et al (1974b) reported that solutions containing prostaglandins E1(PGE1) injected under the skin overlying the calvarium of adult rats stimulated rapid resorption of the bone, indicating that prostaglandins also have the capacity to induce bone resorption in vivo.

Glucocorticoids and periodontal tissues Applebaum and Seelig(1955) observed microscopic changes in the teeth and the periodontal ligaments of rats after adrenalectomy or cortisone treats supporting bone was lost from molars and a detailed study with a high magnification revealed abnormal changes in alveolar bone of rats were given cortisol.

The effect of prednisolone therapy upon gingival inflammation and periodontal bone loss have been studied in groups of patient suffering from multiple sclerosis who had been in the corticosteroid therapy upto four years (Safkhan and Knuttila 1984). Comparison were made between the group of patients who had suffered from the neurological problems but not receiving steroids and healthy controls. There was no differences in the frequency and severity of periodontal disease between the groups and it was concluded that corticosteroid therapy over 1-4 years had no influence on the measures of periodontal disease in patients suffering from neurological disorder.

\section{Non-Steroidal Anti Inflammatory Drugs}

The NSAID are heterogeneous group of compounds whose analgesic, antipyretic and anti inflammatory result from pharmacological mechanisms that are different from these of anti inflammatory steroids and opioid analgesics(21). 
Role of prostaglandins in periodontal disease (22)Two main lines of research have supported the hypothesis that prostaglandins and other arachidonic acid metabolites are important in the pathogenesis of periodontal disease.

Offenbacher et al (1984) found increasing levels of prostaglandins in crevicular fluid in patients with periodontitis and PGE2 levels were 3 fold higher in patients with juvenile periodontitis as compared to patients to patients with adult periodontitis

Golub et al (1990) studied studied the effects of low dose doxycycline therapy on the gingival crevicular fluid in human subjects. 2 Week regimen of these capsules ( each containing 20 or $30 \mathrm{mg}$ of the drug ) significantly reduced the excess host collagenase activity in extracts of inflamed gingival tissues. This effect was not reduced in placebo, treated patients who had adult periodontitis.

Bisphosphonates: Bisphosphonates are synthetic compound that are taken up preferentially by the skeleton and suppress the osteoclast mediated bone resorption. one amino hydroxyl butylidene bisphosphonate(AB) has been shown to inhibit active bone resorption without interfering the bone formation

\section{Conclusion}

Regular home care by the patient in addition to professional removal of subgingival plaque is generally very effective in controlling most inflammatory periodontal diseases. When disease does recur, despite frequent recall, it can usually be attributed to lack of sufficient supragingival and subgingival plaque control or to other risk factors that influence host response, such as diabetes or smoking.

It should be noted, however, that, to date, no home care products or devices currently available can completely control or eliminate the pathogenic plaques associated with periodontal diseases for extended periods of time. Daily home care and frequent recall are still paramount for long-term success.

Thus the goal of periodontal therapy should include, root debridement, anti-microbial therapy and host modulating drugs to arrest the progress of the disease.

\section{References}

[1] Marsh PD, Bradshaw DJ. Dental Plaque as a Biofilm. J Industrial Microbiology 1995; 15(3): 169-175.

[2] Coghlan A.Slime City. New Scientist 1996; 2045:32-36.

[3] Kaldahl WB, Kalkwarf KL, Patil KD. A review of longitudinal studies that compared periodontal therapies. J Periodontol 1994;65(7):731-33.

[4] Kaldahl,WB,Kalkwarf KL,Patil KD, Molvar MP, Dyer JK. Long-term evaluation of periodontal therapy: 1. Response to 4 therapeutic modalities. J Periodontol 1996; 67(2):93-102.

[5] Van Winkelhoff AJ, Rams TE, Slots J. Systemic antibiotic therapy in Periodontics. Periodontol s2000. 10: 45-78.

[6] Listgarten MA, Lindhe J,Hellden L.Effect of tetracycline and/or scaling on human periodontal disease. Clinical, microbiological, and histopathological observations. J Clin Periodontol 1978:5:256-71.

[7] Hellden LB, Listgarten MA, Lindhe J. The effect of tetracycline and /orScaling on human periodontal disease. J Clin Periodontol 1979:6:222-30.

[8] Walker CB,Tyler KT,Low SB,King CJ. Pencillin-degrading enzyme in sites associated with adult periodontitis. Oral Microbiol Immunol 1987:2:129-131.

[9] Magnusson I,Clark WB,Low SB,Maruniak J,Mark RG,Walker CB. Effect of non surgical periodontal therapy combined with adjunctive antibiotics in subjects with refractory periodontal disease. J Clin Periodontol 1989:16:647-653.

[10] Walker CB, Niebloom TA,Gordon JM,Socransky SS. In vitro susceptibilities of bacteria from human periodontal pockets to 13 antimicrobial agents. In: Nelson JD,Grassi C.Current chaemotherapy and infectious disease, Vol1. Washington, D.C:American society of microbiology, 1980: 508-511

[11] Britt MR,Pohlod DJ.Serum and crevicular fluid concentration after a single dose of metronidazole. J periodontol 1986:57:104-107.

[12] Van Oosten MA,Notten FJ,Mikx FH.Metronidazole concentration in human plasma,saliva and gingival crevicular fluid after a single dose.J Dent Res 1986:65(12):1420-23.

[13] Kienfelder JW,Muller RF,Lange DE.Antibiotic susceptibility of putative periodontal pathogens in advanced periodontitis patients. J Clin Periodontol 1999:26:347-351

[14] Loesche WJ, Giodano JR,Hujoel P,Schwarcz J,Smith BA. Metronidazole in Periodontitis.III. Reduced need for surgery. J Clin Periodontol 1992: 19: 103-112

[15] Loesche WJ,Schmidt E, Smith BA,Morrison EC, Caffesse R,Hujoel P.Metronidazole in Periodontitis II. Effect upon treatment needs. J Periodontol 1991:62:247-257.

[16] Quirynen M, TeughelsW, De Soete M, Van Steenberghe D.Topical antiseptics and antibiotics in the intial therapy of chronic adult periodontitis: microbiological aspects Periodontol 2000. 2002:28:72-90.

[17] Quirynen M, TeughelsW, De Soete M, Van Steenberghe D.Topical antiseptics and antibiotics in the intial therapy of chronic adult periodontitis: microbiological aspects Periodontol 2000. 2002:28:72-90.

[18] Greenstein G.Povidone-iodine's effects and role in the managements of periodontal disease: A review. J Periodontol 1999:70:13971405

[19] Diane Cummins. Vehicles: how to deliver the goods. Periodontol 2000 1997;15: 84-99

[20] Nyman S,Schroeder HE,Lindhe J: Suppression of inflammation and bone resorption by indomethacin during experimental periodontitis in dogs. J Periodontol 1979;50L450-61

[21] Johnson RH, Armitage GC, Fransico C,Page RC: Assessment of non steroidal anti-inflammatory drug, Naprosyn in the treatment of gingivitis J Periodont Res 1990,25:230-235.

[22] Kazyuki Noguchi and Isikawa: The role of cylooxygenase -2 and PGE 2 in periodontal disease .Periodontol 2000,Vol-43,2007,87100. 